\title{
Charles Huber, voyageur en Arabie : sur deux manuscrits de poésie bédouine de la Bibliothèque nationale et universitaire de Strasbourg
}

\section{Marcel Kurpershoek et Claude Lorentz}

Traducteur : Coline Godard et Claude Lorentz

\section{(2) OpenEdition}

Journals

Édition électronique

URL : http://journals.openedition.org/rbnu/316

DOI : 10.4000/rbnu.316

ISSN : 2679-6104

\section{Éditeur}

Bibliothèque nationale et universitaire de Strasbourg

\section{Édition imprimée}

Date de publication : 23 mai 2018

Pagination : 100-111

ISSN : 2109-2761

\section{Référence électronique}

Marcel Kurpershoek et Claude Lorentz, "Charles Huber, voyageur en Arabie : sur deux manuscrits de poésie bédouine de la Bibliothèque nationale et universitaire de Strasbourg », La Revue de la BNU [En ligne], 17 | 2018, mis en ligne le 05 septembre 2018, consulté le 10 décembre 2020. URL : http:// journals.openedition.org/rbnu/316; DOI : https://doi.org/10.4000/rbnu.316

La Revue de la BNU est mise à disposition selon les termes de la Licence Creative Commons Attribution - Pas d'Utilisation Commerciale - Partage dans les Mêmes Conditions 4.0 International. 
VARIA

\section{Charles Huber, voyageur en Arabie :}

\section{sur deux manuscrits de poésie bédouine de la Bibliothèque nationale et universitaire de Strasbourg}

PAR MARCEL KURPERSHOEK

AVEC LA COLLABORATION DE CLAUDE LORENTZ

(TRADUCTION FRANÇAISE COLINE GODARD, CLAUDE LORENTZ)

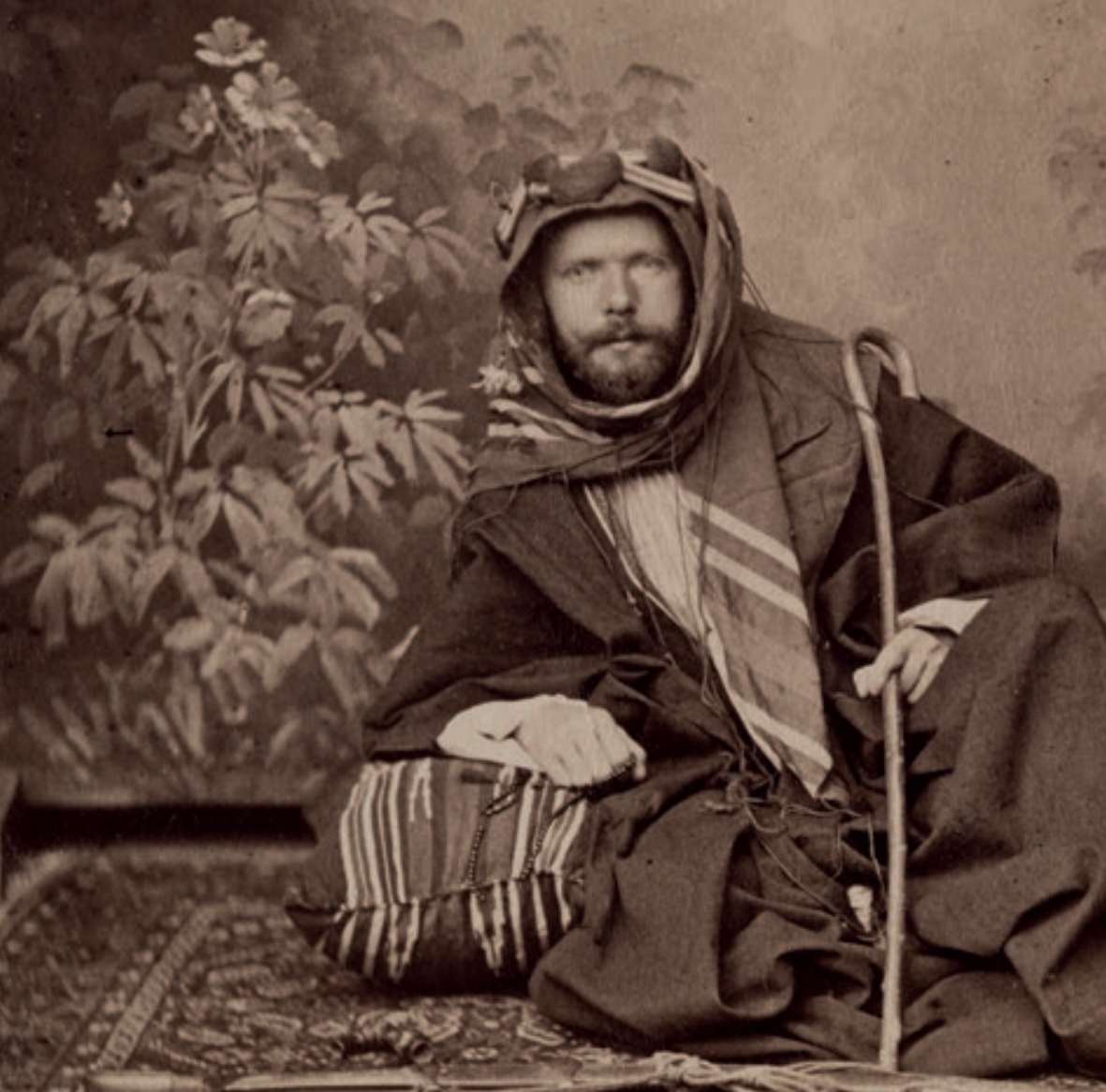




\section{La poésie arabe nabaṭi : cadre général}

La Bibliothèque nationale et universitaire de Strasbourg conserve deux manuscrits de poésie bédouine acquis par l'explorateur Charles Huber (1847-1884) lors de ses missions en Arabie, en 1878 et en 1883-1884 ${ }^{1}$. Il s'agit des deux plus anciens manuscrits connus de poèmes écrits dans la langue vernaculaire de l'Arabie, plus précisément de la province de Nadj située dans la partie centrale de la péninsule arabique. Cette province se trouve au cœur d'un vaste réseau culturel tribal qui s'étend du Yémen et du territoire d'Oman au sud, à la Mésopotamie et au désert syrien, jusqu'aux confins des hauts plateaux d'Anatolie (dans l'actuelle Turquie) au nord.

Jusqu'au $20^{\mathrm{e}}$ siècle, dans cette région, seuls les membres de la classe religieuse et de l'élite des villes étaient lettrés. Pour la vaste majorité de la population, la parole restait le seul moyen de communication. Tout comme à l'aube de la civilisation arabe, avant et après le début de l'ère islamique, la poésie s'imposait comme un moyen classique de formaliser davantage les relations sociales. En raison de conditions économiques ayant peu évolué et de la position isolée de la province de Nadj, entourée de toute part par le désert, cette tradition culturelle a perduré sans interruption pendant près de 1500 ans. Durant mes recherches en Arabie saoudite dans les années 1980 et 1990, j'ai constaté que cette tradition subsistait parmi les anciens, poètes tribaux illettrés et récitants ${ }^{2}$. Cette forme de poésie fait figure de source principale, en dehors de la religion, concernant la vie et la culture en Arabie centrale lors des quatre siècles passés ${ }^{3}$.

Ce genre poétique est aujourd'hui connu, dans son acception générale, sous le nom de poésie nabați. Ce terme fait l'objet de plusieurs interprétations quant à ses origines, parfois rattachées au peuple ancien nabatéen, une théorie pourtant peu vraisemblable. Par sa structure, son imagerie et ses thèmes, mais aussi sa terminologie, sa métrique et ses rythmes, cette forme poétique rappelle plutôt les premiers poèmes arabes classiques. La langue, pourtant, s'éloigne de l'arabe classique comme de l'arabe moderne actuel. Ces variations linguistiques sont liées à l'évolution du langage oral et se reflètent dans la prosodie et d'autres éléments propres à la poésie nabatị. Il serait cependant inexact de ne considérer les vers nabați que comme une poésie simplement écrite dans les langues et les dialectes locaux. Il s'agit plutôt d'un idiome poétique spécifique, influencé à la fois par la tradition classique ancienne et le jargon quotidien utilisé en Arabie au cours des derniers siècles.

Si la poésie nabați, trésor du patrimoine arabe immatériel de cette période et expression d'une culture vivante, revêt une importance particulière, elle n'a jusqu'à récemment que peu attiré l'attention des universitaires, contrairement à la littérature arabe classique. Aujourd'hui encore, elle est plus souvent considérée comme un simple élément complémentaire à d'autres domaines que comme une clé de lecture de cette vaste étendue géographique et culturelle qu'est l'Arabie. Ceci s'explique par diverses raisons. Dans les pays concernés, notamment, cette forme de poésie n'est pas officiellement reconnue. Dirigeants et autorités religieuses en rejettent en effet le langage et le contenu, mésestimés car non conformes aux structures officielles. Aux traditionnels viviers de culture arabe comme Le Caire, le Levant et Bagdad, la poésie nabați inspire le dédain. Elle est perçue comme le dernier vestige d'une culture bédouine arriérée et ignorante, et non comme la forme d'expression artistique d'une culture riche, ancienne, aux racines profondément ancrées. Le manque d'intérêt relatif des chercheurs occidentaux pour la poésie nabați découle principalement de ces préjugés arabes et islamiques. Au cours des dernières décennies, pourtant, cette vision a évolué. Les travaux du chercheur saoudien Saad Sowayan (Nabati Poetry, paru en 1985 et suivi de nombreux autres ouvrages) ont permis cette transition et éveillé l'attention d'autres intellectuels ${ }^{4}$.

Ceci confère une importance nouvelle aux deux manuscrits de poésie nabați acquis par Charles Huber au $19^{\mathrm{e}}$ siècle et explique également pourquoi aucun manuscrit plus ancien n'est connu à ce jour. Une autre difficulté se pose : la plupart des manuscrits de ce type dont nous disposons est clairement

(Page de gauche) Portrait de Charles Huber assis, en voyageur arabe, vers 1880, photogr., pos. montée sur carton, 9,8 x 15,3 cm (coll. BNU) 
l'œuvre de scribes seulement partiellement lettrés, peu coutumiers des règles et de l'orthographe de la langue arabe formelle. Ils rédigeaient les poèmes phonétiquement en lettres arabes, en fonction de leurs connaissances et souvent dans une écriture très médiocre et à peine lisible. Par conséquent, ces manuscrits servaient surtout de supports à des récitants déjà ancrés dans cette culture orale et n'avaient pas vocation à susciter l'intérêt des non-initiés. Ils ne contiennent bien évidemment aucune note explicative ni aucun outil permettant à ces non-initiés de mieux en comprendre le contenu et le contexte. À cet égard, les manuscrits nabați sont aux lecteurs ce qu'une terre vierge et inconnue est aux voyageurs, et il est certain, par exemple, que Charles Huber n'aurait jamais été en mesure de déchiffrer ces textes acquis après sa mort par la Kaiserliche Universitäts- und Landesbibliothek zu Strassburg ${ }^{5}$.

\section{Les manuscrits Huber de la BNU : une anthologie de la poésie nabaṭi}

Les écrits publiés par Huber ne dévoilent pas comment, où et à qui les manuscrits furent achetés. Ces informations apparaissent peut-être dans des documents privés ou autres que je n'ai pas eu l'occasion de consulter ${ }^{6}$. L'inscription allemande « Beduinen Lieder " ( Chants bédouins ») orne la première page vierge de l'un des manuscrits ${ }^{7}$. Si cette description, au vu des hypothèses précédentes, est compréhensible, elle n'en est pas moins inexacte. En réalité, ni la totalité ni même la majorité des poètes présents dans ce manuscrit ne peuvent être qualifiés de «bédouins ». La plupart d'entre eux venaient de petites villes des oasis de Nadj et des environs. Ces poèmes, en outre, étaient surtout récités, même si certains d'entre eux étaient effectivement chantés au rythme du ribābih, un instrument à une corde. Les poèmes chantés sont ordinairement courts (sept lignes en moyenne) ${ }^{8}$. Or ceux contenus dans ces manuscrits sont plus longs.

$\mathrm{Au}$ moment où Huber les a acquis, ces manuscrits étaient sans nul doute récents. En effet, l'un des poètes, Mḥammad al- 'Abdallah al-Qāḍi, riche et célèbre érudit de la ville d'Unaizah (province d'alQașīm), est mort en 1868, soit à peine plus de dix ans avant la visite du Français. Mbārak ibn Mwēm, poète membre du groupe al-Wudā īn de la confédération al-Dawāsir, meurt quant à lui en 1864. Le poète 'Ubayd ibn Rashīd, surnommé « le loup ", s'éteint à son tour l'année suivante. Avec son frère Abdallah, il avait fondé à Haïl la dynastie $\mathrm{Al}$ Rachid, qui éclipsa les princes $\mathrm{Al}$ Saoud à Riyad. Les poèmes les plus anciens de ces manuscrits, comme ceux écrits par Quṭn ibn Quṭn, al- 'Ulaymi et Barakāt al-Sharīf, remontent au $17^{\mathrm{e}}$ siècle. C'est par exemple le cas de l'un d'entre eux, présent dans un autre manuscrit et écrit en l'honneur de Barakāt al-Sharīf en 1674, l'an 1085 de l'Hégire ${ }^{9}$. Rumaizān ibn Ghashshām, un autre de ces poètes, meurt en $1680^{10}$. Ces textes anciens furent sans doute copiés de manuscrits dont nous n'avons pas connaissance. Ils furent nombreux à être brûlés en Arabie lors de la conquête, portée par un fort esprit wahhabite, d'une grande partie du territoire par l'Ikhwān. Cette armée, constituée de membres de tribus bédouines, forma plus tard les troupes d'assaut du roi 'Abdul 'Azīz. En tout état de cause, il est fort peu probable que ces poèmes aient perduré pendant des siècles simplement par transmission orale.

Dans l'ensemble, les poètes présents dans les manuscrits de Huber sont des noms célèbres et récurrents dans les recueils plus tardifs de poésie nabați. De par leur nature, les textes poétiques qui ont circulé à l'oral comme à l'écrit présentent au fil du temps des variations considérables en termes de formulation et d'ordre des vers notamment. En l'absence de l'auteur ou d'une source proche faisant autorité, il devient impossible de déterminer avec certitude l'authenticité de la formulation originale. Par contre, un poème connu reste facilement identifiable, même avec des vers rarement identiques dans différentes versions ${ }^{11}$.

C'est le cas d'un poème de Mbārak ibn Mwēm dont j'ai relevé une version en 1989 à Wādi al-Dawāsir, dans le sud de la province de Nadj, à environ $700 \mathrm{~km}$ de la capitale, Riyad, très loin de l'endroit où Huber acheta les manuscrits. Ce poème est resté populaire par ses vers sur les thèmes de la sagesse et des grands principes de conduite vertueuse dans la société de $\mathrm{Nadj}^{12}$. Il se trouve également dans l'un des manuscrits de Charles Huber. Mbārak appartenait à la tribu d'al-Wudā īn, de la confédération Dawāsir. Il s'agit de la tribu dirigeante des groupes 
sédentaires (de l'arabe « ḥaḍar », un terme dont l'étymologie est liée à « ḥaḍārah », « civilisation »), par opposition à d'autres branches de la confédération liées à des tribus bédouines ("al-bādiyah », « ceux qui vivent dans le désert »). Par principe, les habitants de maisons en terre peuplant villages et petites villes étaient considérés (et se considéraient eux-même) comme davantage " religieux ", c'està-dire assidus dans l'exercice de leurs obligations religieuses, et mieux éduqués que les Bédouins à la bienséance islamique. Même à Wādi al-Dawāsir, où sédentaires et Bédouins se côtoyaient, les différences de perception et de comportement étaient manifestes.

La version du poème d'Ibn Mwēm que j'ai relevée comptait 38 vers, contre 49 dans celle du manuscrit de Huber. Dans d'autres versions, ce nombre varie de 43 à 56 . L'ordre des vers est également totalement différent, de même que la plupart des formulations. Malgré cela, le poème est identifiable au premier regard et sans l'ombre d'un doute. Cela s'explique en partie par le fait que les vers sur la sagesse ne sont liés à aucun développement thématique et se trouvent donc facilement interchangeables. Ils sont d'ailleurs souvent comparés à des perles sur un fil, que le poète déplace à sa guise. Ibn Mwēm a beaucoup voyagé, et ses vers étaient assurément connus dans le Golfe où de nombreux membres de Dawāsir travaillaient dans l'industrie perlière. Grâce à sa notoriété, ce poème fut ainsi inclus dans l'anthologie des manuscrits de Charles Huber. Il est généralement admis, cependant, que les versions les plus fiables d'un texte sont souvent trouvées à proximité du lieu d'origine du poète et parmi les membres de sa tribu et de sa famille.

Même les poèmes trouvés à des périodes proches de l'original, mais à des distances géographiques élevées, peuvent s'avérer moins fiables que certaines versions plus tardives mais préservées parmi les proches de l'auteur, aussi bien sous forme écrite qu'orale. C'est notamment le cas du célèbre poème écrit par Dandan al-Fhēm, de la tribu des Shammar, pour le chef suprême des Shammar d'al-Jarba. À cette époque, la tribu avait migré au nord de l'Irak suite à une défaite face aux troupes Wahābi des princes Al Saoud. Là encore, quasiment aucun vers ne correspond dans la version du manuscrit de Huber et dans une autre ${ }^{13}$. Comme évoqué précédemment, il est impossible de considérer a priori la version de Huber comme plus proche de l'original, en raison de sa date ancienne. Des erreurs de transmission peuvent être commises dès le début puis répétées par la suite.

La date du manuscrit de Charles Huber pourrait s'avérer importante et permettre d'accéder à une version proche du poème original mais pour en être certain, il faudrait pouvoir effectuer un travail de comparaison précis avec d'autres versions connues.

Les chercheurs se sont jusqu'à présent attachés à poser les jalons nécessaires à des études approfondies et à définir les paramètres d'un vaste travail académique, là où l'exploration n'avait pas encore réalisé de percée. Pour les manuscrits de Charles Huber, contrairement à la poésie classique arabe, il n'existe aucune édition commentée présentant une vue d'ensemble des différentes versions. Cette tâche incombera aux futures générations de chercheurs. Il n'est donc pas improbable que les manuscrits de cet explorateur, après un sommeil de plus d'un siècle, commencent à éveiller un intérêt nouveau dans les temps à venir.

\section{Quelques remarques à propos des carnets} de voyage de Charles Huber en Arabie

Jusqu'à présent, mes recherches sur les origines des manuscrits de Huber sont restées relativement infructueuses. Les carnets et notes de ses voyages en Arabie présentent cependant un intérêt majeur. Lors de mes voyages d'étude à Hail, la capitale du nord de l'Arabie saoudite, et dans la région du désert du Nefoud, j'ai découvert que le travail de Huber et d'autres explorateurs européens du $19^{\mathrm{e}}$ et du début du $20^{\mathrm{e}}$ siècles était en grande partie identifiable ${ }^{14}$.

Lors de ses séjours en Arabie, Charles Huber était bien conscient de marcher dans les pas d'autres explorateurs européens arrivés avant lui. Il évoque d'ailleurs souvent Georg August Wallin (1811-1852), Charles Montagu Doughty (1843-1926) et le couple Wilfrid Scawen Blunt (1840-1922) et Lady Anne Blunt (1837-1917). Il mentionne des personnalités locales rencontrées lors de ses séjours et également connues de ces explorateurs. Il va même jusqu'à comparer l'accueil chaleureux qu'il a pu recevoir avec le rejet irrévérencieux subi par Doughty lors de son arrivée. 
Avant d'embarquer pour son premier voyage en 1879, Huber présente dans une note son projet, ses objectifs et les moyens envisagés pour obtenir soutiens et financements ${ }^{15}$. Le premier de ses objectifs porte sur son désir de « saisir toute occasion de rechercher ruines et monuments anciens pour les décrire et en copier les inscriptions » et de "ramasser des matériaux anthropologiques et géographiques ». Mais les manuscrits font déjà leur apparition : « Je recueillerai aussi tous les manuscrits que je pourrai me procurer », indique-t-il.

Comme d'autres visiteurs de l'arrière-pays arabe exclusivement musulman, Huber envisage d'adopter une identité musulmane et se prépare à cette transformation religieuse et culturelle en séjournant dans les régions plus tempérées de l'Égype et du Levant. " [Avant le départ je veux vivre] pendant le temps nécessaire en Égypte pour me dépouiller de l'Européen et le faire complètement oublier ", écrit-il. Il compte pour cela sur ses contacts en Égypte et en Syrie. Il fait face à une difficulté de taille quant au volet anthropologique de sa mission qui consistera à mesurer des crânes humains, face à un groupe de musulmans méfiants. Il estime alors que sa capacité à réaliser des interventions chirurgicales légères lui permettra d'effectuer ces mesures que les patients ne seront pas en position de refuser. Il évoque, en dernier lieu, son intention de vérifier l'information selon laquelle « quelques descendants des Arabes contemporains de Mohammad " vivent dans les environs de Médine. Il s'agit pour Huber d'« Arabes primitifs " ("Uraraber») des tribus 'Aws et Khazraj, pratiquants chiites « réfractaires à l'absorption par leurs voisins [sunnites]». Cette note est complétée par une citation de Richard Strachey définissant le voyageur comme « le prisonnier de la science » qui doit fournir les informations nécessaires à d'autres chercheurs dans son pays d'origine.
Pour sa seconde expédition en 1883-1884, Charles Huber est accompagné de l'orientaliste allemand Julius Euting (1839-1913), premier bibliothécaire puis directeur, de 1900 à 1909, de la Kaiserliche Universitäts- und Landesbibliothek de Strasbourg, l'ancêtre de l'actuelle Bibliothèque nationale et universitaire ${ }^{16}$. Contrairement à Euting qui publia son Carnet de voyage en Arabie centrale ${ }^{17}$, Huber ne cherchait pas à transposer ses expériences dans un récit littéraire cohérent visant le grand public, évoquant l'aventure, dressant un tableau saisissant du peuple arabe et des relations qu'il avait pu avoir avec lui. Au contraire, ses deux publications (Journal d'un voyage en Arabie centrale, 1883-1884 et un article dans le Bulletin de la Société de géographie, «Voyage dans l'Arabie centrale ${ }^{18}$ ) s'apparentent davantage à des journaux de bord rapportant minutieusement chaque action, chaque observation et information factuelle que l'explorateur a pu collecter dans le cadre de son programme, comme il le souligne dans ses notes.

Tous les jours, Charles Huber s'applique à noter l'heure exacte du départ le matin, les distances pacourues, le noms des endroits, les différents types de sols et de formations géologiques, les noms des arbres, plantes et herbes ainsi que leurs noms arabes, les noms des tribus, sous-tribus, chefs et autres personnes rencontrées. L'une de ses tâches principales consiste à copier les inscriptions anciennes pré-arabes trouvées sur des roches ou des tablettes en pierre. Euting rapporte que son compagnon était constamment occupé à prendre des mesures et à observer les étoiles avec son théodolite afin de retracer de manière exacte les cartes de son voyage. La ténacité et la capacité de résistance de Charles Huber semblent sans limites. En traversant al-Qașīm au cœur de l'été, il dut faire face par $50^{\circ}$ à « l'air lourd comme du plomb ", avec « la tête et tout le corps enveloppés comme pour un voyage au pôle nord, afin

\footnotetext{
(Page de droite) Le manuscrit MS.4.271 de la BNU contient trois poèmes de Ḥmēdān al-Shwē ir comptant parmi les plus importants de cet auteur du $18^{\mathrm{e}}$ siècle qui vivait dans la région du Nadj, en Arabie centrale, peu avant l'hégémonie du mouvement wahhabite. Ḥmēdān al-Shwēe ir était un maître de la satire, connu pour son humour galant, son autodérision et ses vers incisifs. Il se révèlait particulièrement acerbe dans ses critiques de la société et de ses mœurs.

Dans le poème 21 ici présenté, le poète offre ses excuses au prince le plus puissant de son temps dans cette région, 'Abdallah ibn $\mathrm{Mu}$ 'ammar qu'il aurait insulté, ce qu'il nie. Ce texte s'apparente aux fameux poèmes d'excuses du poète pré-islamique al-Nābighah al-Dhubyān̄̄ adressés au Lakhmide roi de Ḥīra, Nu 'mān ibn Mundhir.

Traduction en anglais dans Marcel Kurpershoek (ed. and tr.), Arabian Satire. Poetry from 18th Century Najd. Himēdān al-Shwē ir, New York, New York University Press, 2017, p. 80.
} 


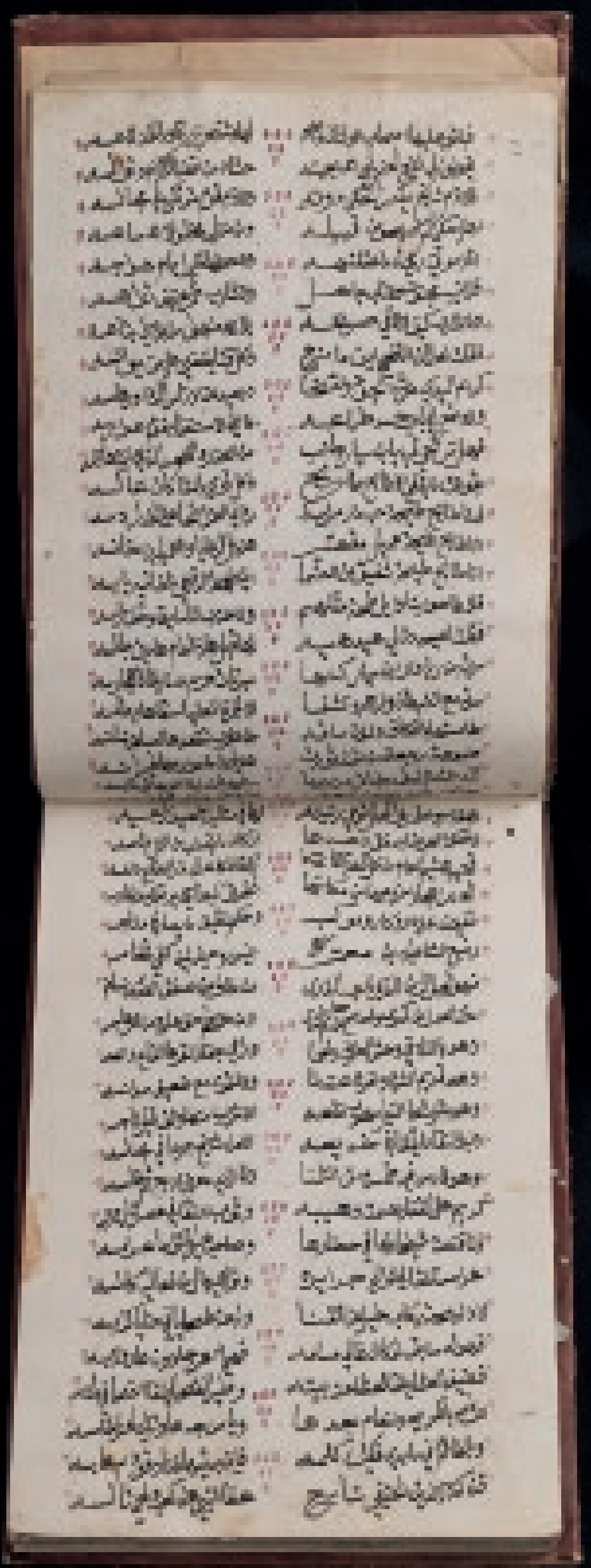


de les soustraire à l'action du soleil et du vent brûlants », en se sentant «la tête prise comme dans un cercle de fer $"{ }^{19}$.

Le dirigeant de Haïl, Muḥammad ibn Rashīd, dont l'influence ne cesse de croître, semble tenir Huber en haute estime. L'explorateur a l'avantage de disposer d'une pleine autorisation du gouvernement français pour ses voyages. Lorsqu'il compare l'accueil chaleureux que lui réserve le gouverneur de la ville de Khaybar au rejet subi par son contemporain, le voyageur anglais Charles Doughty ${ }^{20}$, face à ce même dirigeant, Huber note que contrairement au Britannique, il disposait d'un firman, un édit officiel émis par le sultan ottoman. Huber comprend que les chances de réussite d'une mission en Arabie reposent en partie sur la capacité des explorateurs à bien se présenter. Ibn Rashīd, en retour, demande à Huber de faire savoir aux autorités turques qu'il se considère comme un serviteur dévoué de l'État ottoman.

Les deux hommes développent une relation amicale au fil des visites fréquentes de Huber à la cour. L'explorateur note les traits de caractère d'Ibn Rashīd : sa vigilance permanente, sa fermeté dans son gouvernement et son sens de la diplomatie, sa connaissance approfondie de la géographie et de la société arabes, sa capacité à gérer les tribus, mais également sa curiosité, l'aspect léger et jovial de sa personnalité. Le prince évoque par exemple une visite de Wilfrid et Lady Anne Blunt, fasciné par le fait que le premier ne pouvait boire son café qu'une fois sa femme servie, la laissait passer avant lui à chaque occasion en se contentant d'être au second plan. Ibn Rashīd se disait déconcerté de voir avec quelle facilité Lady Anne pouvait monter à cheval. «En est-il ainsi chez tous les chrétiens ? ", demande-t-il à Huber.

L'explorateur rapporte à plusieurs reprises la préoccupation de l'émir pour son confort et sa sécurité pour les voyages prévus, en particulier lors de son passage vers al-Qașīm, à mi-distance environ sur la route de Riyad, ainsi que vers Djeddah. Ce port de la mer Rouge est une porte d'entrée vers La Mecque et abrite les missions diplomatiques de la France et d'autres pays. En 1884, Huber quitte Haïl pour Djeddah, armé d'un sauf-conduit émis par le prince. Mais la tribu 'Utaybah ('Tēbah), qui contrôle une grande partie de ce territoire, n'est pas entière- ment sous l'autorité d'Ibn Rashīd. Les raids lancés par le prince sur leur territoire et le vol de chameaux, chevaux et d'autres possessions ne jouent sans doute pas en sa faveur.

Huber rejoint finalement La Mecque après une expérience terrible qui le laissera ébranlé, avec son guide du clan Shiyābīn de la tribu 'Utaybah ${ }^{21}$. Dans ses dernières notes datées du 20 juin à Djeddah, Huber informe le secrétaire général de la Société de géographie, Charles Maunoir, de sa volonté de rester seulement quelques jours dans la ville avant de rejoindre Hail puis Bagdad pour rentrer chez lui, se sentant gravement menacé par des éléments de la tribu 'Utaybah ; « tous m'attendent pour me piller et me tuer », explique-t-il à Maunoir. Il souhaite envoyer ses copies d'inscriptions et ses carnets, et précise : « je risque beaucoup en retournant d'ici au désert, je le sais... En vous envoyant mon journal, au moins si je suis tué tout ne sera pas perdu ». L'explorateur demande à Maunoir de remettre ces documents à Ernest Renan, l'éminent philosophe et spécialiste des langues anciennes du Moyen-Orient. Il est déjà démoralisé à l'idée de n'avoir pas reçu la dernière lettre de Maunoir qui lui aurait « donné un peu de cœur ». " J'aurais aimé vous parler un peu de mes courses mais votre silence me glace. Si je suis aussi en disgrâce près de vous, comme je le suis au ministère [de l'Éducation nationale, l'autre soutien financier du voyageur], je n'oublie pas ce que je vous dois... ».

À peine un mois plus tard, le 29 juillet 1884, Charles Huber est pillé et tué de sang-froid par ses deux guides à Rābigh, lors du trajet entre Djeddah et Haill. Son serviteur Mạ̣mūd le découvre pendant la prière des deux tueurs. Il est alors autorisé à porter la funeste nouvelle auprès du consul de France à Djeddah, Félix de Lostalot $^{22}$.

\section{Charles Huber et Julius Euting : entre coopération et concurrence}

Pour conclure, évoquons en quelques mots la relation entre Charles Huber et son compagnon de voyage Julius Euting. Étonnement, les journaux publiés par Huber ne font aucune mention de ce dernier. Ses écrits ne précisent pas même le fait que les deux hommes voyagent ensemble. Euting, 
a contrario, évoque largement la présence de son compagnon dans les deux volumes de son Tagebuch einer Reise in Inner-Arabien. L'index comprend même une sous-rubrique sur " la perfidie d'Huber » envers Euting. Il s'agit d'un ajout de l'éditeur Enno Littmann, qui a également introduit au nom d'Euting plusieurs notes accusant Huber d'avoir délibérément manœuvré contre lui à son insu et d'avoir nui à la réputation de son compagnon auprès du prince de Haill.

Les deux hommes partent ensemble vers l'ouest afin de copier des inscriptions nabatéennes, entre autres, à Taima, Khaybar, al- 'Ula et Madā'in Șāliḥ. À ce moment, Huber prend de court son compagnon en lui annonçant qu'Ibn Rashīd ne l'autorise pas à revenir à Haïl et que leurs chemins doivent alors se séparer. Ils se partagent leurs biens et, après avoir donné son arme à Huber, Euting rejoint la ville portuaire de la mer Rouge al-Wajh, d'où il finit par regagner l'Europe et Strasbourg.

Euting soutient que Charles Huber l'accompagne dans ses voyages, et non l'inverse. Il écrit également qu'il a lui-même accepté de choisir au moins une pierre contenant des inscriptions et que le reste des acquisitions reviendrait à Huber. Euting réclame la stèle de Taima, et note que contrairement à l'accord passé avec Huber, cette stèle se retrouve finalement au musée du Louvre grâce à l'intervention du consul de France à Djeddah, F. de Lostalot.

Dans son journal, Euting n'accuse pas directement Huber de ce retournement de situation. Il reconnaît toutefois que les actions d'Huber ont parfois $\mathrm{pu}$ le laisser perplexe, comme ce fut le cas lorsqu'il rendait visite au prince de manière informelle. Il se demande également pourquoi Huber ne l'a informé qu'au dernier moment de la décision d'Ibn Rashīd de ne pas l'autoriser à revenir à Haill. Cette décision, selon Euting, repose en réalité sur le fait qu'il entretenait de mauvais rapports avec Ḥūd ibn 'Ubayd ar-Rashīd, proche et puissant conseiller du dirigeant. Aux yeux d'Euting, Humūd apparaissait comme un Wahhābi sectaire qui n'avait de cesse de le contrarier et le forçait à céder ses armes à feu puis tour à tour tous ses biens. Les remarques furieuses du voyageur à son égard, selon lui, avaient fini par arriver aux oreilles de Himūd, qui devint alors son ennemi juré à la cour. Les récits de voyages d'Euting avec Huber se terminent par « un adieu cordial».
Il est certain, cependant, que Charles Huber a sa part de responsabilité dans le statut de «persona non grata » d'Euting à la cour et son interdiction de séjour à Haïl. Dans une lettre adressée à son ami le Dr. Weißgerber, il évoque sa satisfaction d'avoir réussi à écarter Euting avec l'aide du prince ${ }^{23}$. Il rapporte également la difficulté du transport de la très lourde stèle de Taima à Haïl et assure qu'il n'épargnera aucun effort pour faire en sorte que cette stèle tombe entre les mains des Français. De son côté, dans des correspondances privées, Euting évoque sans ambages son mécontentement face à l'hypocrisie de son compagnon de voyage ${ }^{24}$.

Le caractère ambigu de la relation entre les deux hommes n'est guère surprenant au vu de la situation politique de l'époque, au lendemain de la guerre franco-prussienne de 1870 qui se solde par la perte de l'Alsace-Lorraine. Euting travaillait pour les institutions allemandes au sein de la récente, et déjà imposante de par ses collections, bibliothèque régionale et universitaire de Strasbourg. Huber était quant à lui issu d'une famille française loyaliste clairement dans le viseur des autorités allemandes. Au retour de sa première expédition en Arabie, Huber doit se présenter à la police tous les lundis, justifier chacune de ses absences et demander des permissions, comme le mentionnent Hélène Lozachmeur et Françoise Briquel-Chatonnet dans leur article sur les deux explorateurs, publié dans la revue Anabases ${ }^{25}$.

Il est donc d'autant plus remarquable de constater que, malgré ces tensions politiques latentes, une certaine coopération entre les chercheurs des deux pays fut toutefois possible. Cette histoire démontre finalement qu'au $19^{\mathrm{e}}$ siècle, les milieux savants pouvaient, dans une certaine mesure, à la fois se concurrencer et collaborer en dépassant les obstacles politiques et sociaux. Ces savants étaient avant tout portés par leur soif de découvertes et de grands progrès au nom de leur intérêt commun pour la science.

Objectivement alliés et partenaires, quoique également concurrents, Huber et Euting partageaient en tout état de cause la volonté, au terme de leurs éprouvantes expéditions, de rejoindre la « civilisation " et de laisser " le désert " derrière eux. Après avoir évoqué son dégoût en se souvenant avoir été forcé de boire de l'eau mélangée à des excréments de 


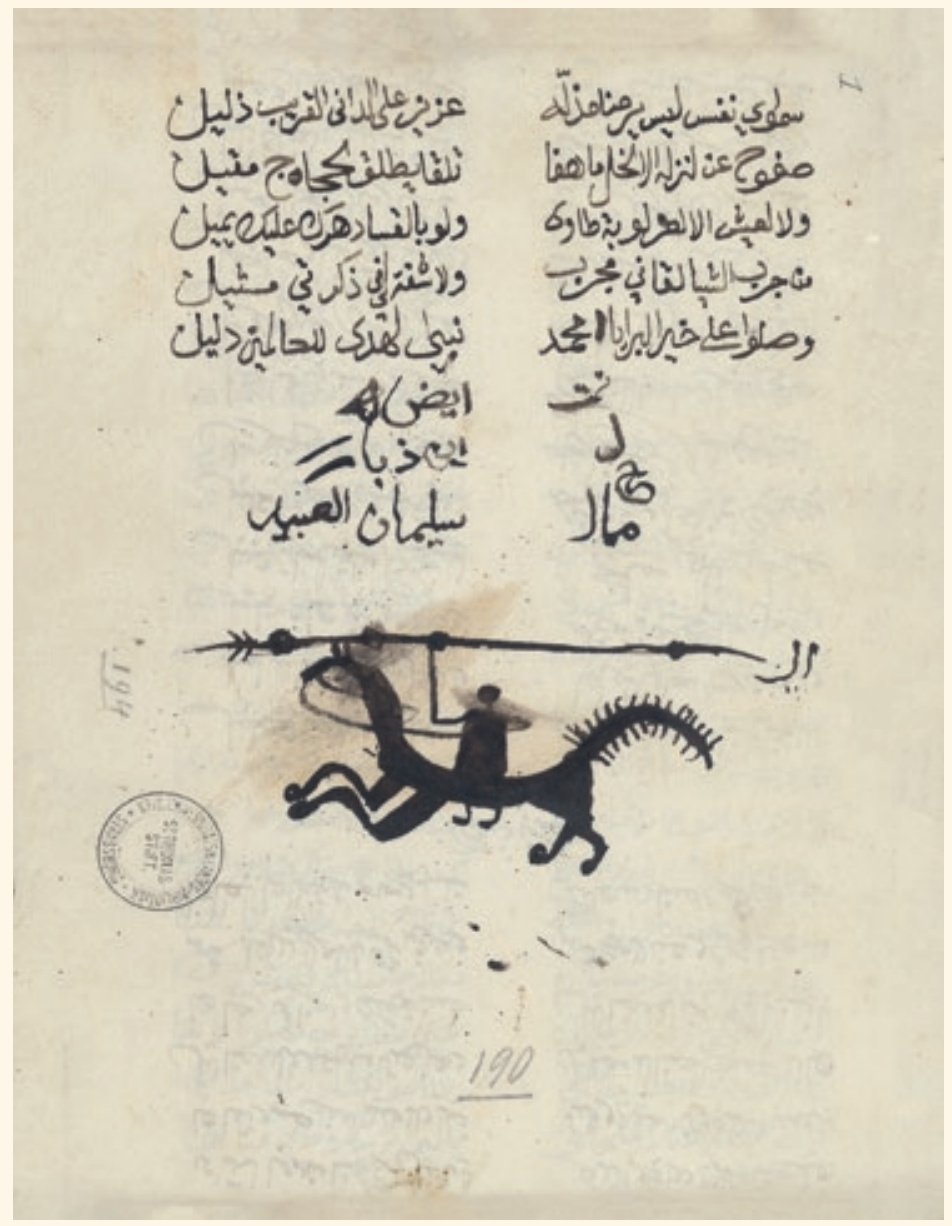

Page du manuscrit MS.4.271, avec le dessin d'un guerrier arabe armé d'une longue lance (coll. BNU)

chameau, Euting rejoint les rives de la mer Rouge à al-Wajh et s'exclame, à la manière des Dix Mille dans l'Anabase de Xénophon : "Quelle joie de rejoindre la civilisation des Égyptiens et des Turcs! », et « Je vous souhaite le meilleur, chers Bédouins. Retournez dans vos tentes du désert où vous êtes chez vous ".

De même, lors de son arrivée à Nedjef (Irak) à la fin de son premier voyage, Charles Huber déclarait, malgré l'épidémie de peste qui sévissait : «[...] la coupole dorée de la mosquée de Nedjef [...] Cette vue donna courage à tout le monde et fit oublier bien des misères passées ». Il ajoutait : " Je m'arrêtai quelques jours à Nedjef et à Kerbela, heureux de goûter de nouveau les douceurs d'une civilisation plus avancée que celle des habitants du désert ${ }^{26}$.
Les manuscrits Huber de la BNU sont consultables sur la bibliothèque numérique Numistral :

- MS. 4.269 : http://www.numistral.fr/ ark:/12148/btvlb10235222b?rk=21459;2

- MS. 4.271 : http://www.numistral.fr/ ark:/12148/btv1b10235223s 


\section{Charles Huber, explorateur de la péninsule arabique}

Charles Huber (1847-1884) est l'un des rares explorateurs français à avoir parcouru la péninsule arabique durant le dernier tiers du $19^{\mathrm{e}}$ siècle, en quête de découvertes tant géographiques, archéologiques, épigraphiques qu'ethnographiques.

Né à Strasbourg, fils d'un modeste cordonnier, il fait des études de chimie et de médecine, occupe divers emplois, avant de laisser libre court à son attirance pour l'Orient, notamment l'Arabie. Après un premier voyage en Afrique du Nord en 1874, il obtient en 1878 une subvention du ministère français de l'Instruction publique pour une mission censée le mener d'Arabie au Tibet, par la Perse et l'Afghanistan. De mai 1879 à avril 1880, il séjourne à Damas où il se familiarise avec les divers dialectes et les mours arabes. Il gagne l'Arabie en mai 1880, atteint Haïl, la capitale du Jebel Shammar, d'où il rayonne dans le Qașīm et vers le Hedjaz. Il y recueille un grand nombre d'inscriptions lihyanites ${ }^{27}$ et coufiques ${ }^{28}$ et précise ou corrige plusieurs points de géographie. Ne pouvant regagner l'Europe par Djeddah et la mer Rouge, il rejoint Bagdad (mars 1881), où il participe à des fouilles sur le site de Babylone, puis Damas (décembre 1881), la France et enfin Strasbourg, sa ville natale, alors la capitale administrative de l'Alsace-Lorraine annexée à l'Empire allemand.

Sur les instances d'Ernest Renan qui dirige la publication du Corpus Inscriptionum Semiticarum, la commission des Missions du ministère de l'Instruction publique le charge bientôt d'une nouvelle expédition en Arabie, à la recherche d'inscriptions qui viendraient compléter les relevés du Britannique Charles Montagu Doughty et de l'Allemand Theodor Nöldeke, ce dernier étant alors professeur à l'université impériale de Strasbourg, la Kaiser-WilhelmsUniversität. C'est à la demande de Nöldeke que Huber se voit adjoindre un compagnon de voyage en la personne de Julius Euting, philologue et épigraphiste, spécialiste des langues sémitiques anciennes et premier bibliothécaire à la Kaiserliche Universitätsund Landesbibliothek. Les deux voyageurs séjournent à partir de l'automne 1883 à Haïl, Taima, Medain Saleh et al-'Ula. Après être parvenu à acquérir la fameuse stèle de Taima, d'importance capitale pour la compréhension des rapports entre l'Arabie et la Mésopotamie au $5^{\mathrm{e}}$ siècle avant notre ère, Huber interrompt sa mission pour se rendre à Djeddah, où il remet ses notes et les estampages de ses inscriptions au consul de France. L'acheminement de la stèle de Taima est quant à lui laissé à la diligence de l'émir de Haïl qui la transmet aux autorités françaises, grâce auxquelles elle entre dans les collections du musée du Louvre. C'est sur la route du retour, à $R a \bar{b}$ igh, sur la mer Rouge, au nord de Djeddah, que Huber est assassiné le 29 juillet 1884, à l'âge de 37 ans, par ses guides arabes qui le dépouillent de ses biens.

La relation du dernier voyage de Charles Huber en Arabie est publiée quelques années plus tard, en 1891, par les soins conjugués de la Société de géographie et de la Société asiatique, et sous les auspices du ministère de l'Instruction publique.

Voyageur instruit comme en témoigne le catalogue de vente de sa bibliothèque riche de 1411 références, audacieux « jusqu'à la témérité ", selon les auteurs de l'introduction de son Journal de voyage en Arabie (E. Renan, Barbier de Meynard et C. Maunoir), Charles Huber laisse une moisson scientifique relative à l'Arabie centrale abondante et polymorphe : déterminations géodésiques, observations hypsométriques, mesures météorologiques, observations géologiques, ethnographiques et archéologiques, documents manuscrits et épigraphiques. Cette documentation est aujourd'hui répartie au sein de diverses institutions françaises, dont le musée du Louvre et la Bibliothèque nationale et universitaire de Strasbourg.

Claude Lorentz 


\section{ÉCRITS DE CHARLES HUBER}

- «Inscriptions recueillies dans l'Arabie centrale, 1878-1882 », in Bulletin de la Société de géographie, 1884, p. 289-303

- « Voyages dans l'Arabie centrale : Hamad, Shammar, Qasim, Hedjaz », in Bulletin de la Société de géographie, 5, 1884, p. 304-363, 468-530, et 6,1885 , p. $92-148$

- Journal d'un voyage en Arabie, 1883-1884, publié par la Société asiatique et la Société de géographie, sous les auspices du ministère de l'Instruction publique, avec atlas, Paris, Imprimerie nationale, 1891

\section{ORIENTATIONS BIBLIOGRAPHIQUES}

- Broc, Numa et Siary, Gérard, Dictionnaire illustré des explorateurs et grands voyageurs français du XIX ${ }^{e}$ siècle, Paris, Éd. du Comité des travaux historiques et scientifiques. II, Asie, 1992, p. 245-246

- Calvet, Yves et Robin, Christian, Arabie heureuse, Arabie déserte. Les antiquités arabiques du musée du Louvre, Paris, Éditions de la Réunion des musées nationaux, 1997

- Lozachmeur, Hélène et Briquel-Chatonnet, Françoise, « Charles Huber und Julius Euting in Arabien nach französischen, auch heute noch nicht veröffentlichten Dokumenten », in Anabases, $n^{\circ} 12,2010$, p. 195-200

- Nouveau dictionnaire de biographie alsacienne, Strasbourg, Fédération des sociétés d'histoire et d'archéologie d'Alsace, $n^{\circ} 17$, p. 1678-1679

- Pouillon, François (éd.), Dictionnaire des orientalistes de langue française, Paris, IISMM, Karthala, 2008, p. 498-499

- Catalogue des livres composant la bibliothèque de feu M. Charles Huber de Strasbourg, membre de la Société de géographie assassiné en voyage d'exploration en Arabie. Vente le 22 juin 1885 et les jours suivants, place Saint-Étienne, Strasbourg, J. Béguin, 1885 (cote BNU : M.200)

\section{NOTES}

1- Poésies des Arabes du désert, parmi lesquelles celles d'alMahadi, Targui ibn Hamid, Zaïd al-Salama, Abd ibn Rashid et d'autres et Poésies des Arabes du désert, recueillies par Huber, et copiées à Hadjil ; de Hamid ibn al-Shouï'ar ; Abd Allah ibn Ma'mar ; Moubarak ibn Omouyam ; Abou-Hamza ; al-Vali ; Barakat al-Sharif; al-Sho'aïbi, contre Barakat al-Sharif, etc. Manuscrits de la Bibliothèque nationale et universitaire de Strasbourg, respectivement cotes MS.4.269 et MS.4.271. Ces manuscrits ont été achetés par la Kaiserliche Universitäts- und Landesbibliothek zu Strassburg (actuelle BNU) lors de la vente aux enchères de la bibliothèque de Charles Huber qui s'est déroulée à Strasbourg le 22 juin 1885 et les jours suivants.

2- Les résultats de ces recherches ont été publiés dans Oral Poetry and Narratives from Central Arabia, Leiden, New York, Brill Publishers, 1994-2005, 5 volumes. Le récit des recherches figure dans Arabia of the Bedouins (traduction anglaise du néérlandais), London, Saqi, 2001, et en traduction arabe : $A l$ Badawī al-Akhīr (The Last Bedouin), 2002.
3- Par exemple, le poète Humaydān al-Shuway'ir, qui vivait dans une petite ville au nord de Riyad dans la seconde moitié du $17^{\mathrm{e}}$ et la première moitié $\mathrm{du} 18^{\mathrm{e}}$ siècle, est une source majeure de connaissances sur la culture et la mentalité populaires avant l'avènement du mouvement de réforme wahhābi, en conjonction avec la montée de la maison de Sa'ūd à partir de 1745 . Il prit une part active aux luttes politiques entre les petites villes et les dirigeants de l'Arabie centrale à cette époque, et plusieurs de ses versets reflètent des proverbes et des dictons populaires qui continuent à être utilisés. À l'époque moderne, sa poésie a fait l'objet d'un débat intellectuel animé et reste très connue. Mon édition et la traduction de ses poèmes figurent dans Marcel Kurpershoek (ed. and tr.), Arabian Satire. Poetry from $18^{\text {th }}$ Century Najd. Hmèedān al-Shwē ir $\mathrm{ir}$ New York, New York University Press, 2017. Trois de ses poèmes parmi les plus importants et les plus longs sont présents dans les manuscrits acquis par Charles Huber.

4- Sowayan, Saad Abdullah, Nabati Poetry, the Oral Poetry of Arabia, University of California Press, 1985

5- Voir supra, note 1.

6- Les archives de Charles Huber sont conservées à la bibliothèque du Cabinet du corpus des inscriptions sémitiques appartenant à l'Académie des Inscriptions et Belles-Lettres, l'une des cinq académies de l'Institut de France. Je n'ai pas encore pu étudier ces papiers.

7- Il s'agit du MS.4.269.

8- Sowayan, Saad Abdullah, Nabati Poetry, the Oral Poetry of Arabia, op. cit., p. 138

9- Sowayan, Saad Abdullah, Al-Shi 'r al-Nabați, dhā'iqat alsha'b wa-sultat al-nass (Nabati Poetry, Popular Taste and the Authority of the Text), 2000, p. 361

10- Ibid., p. 439

11- Cependant, il arrive assez souvent que deux poèmes du même mètre et de la même rime s'emmêlent dans la transmission.

12- Voir Kurpershoek, Marcel, Oral Poetry and Narratives from Central Arabia, vol. 4, A Saudi Tribal History, Honour and Faith in the Traditions of the Dawāsir, op. cit., p. 680-685, p. 700-707

13- 'Abd al-Raḥmān al-Suwaydā', Judhū' wa-furū' (Racines et branches), 2, 1986, p. 43-63. Sur l'histoire de Dandan al-Fhēm et ses poèmes, voir Marcel Kurpershoek, Arabia of the Bedouins, op. cit., p. 134-138.

14- Kurpershoek, Marcel, Arabia of the Bedouins, part 2, Shammar ; l'histoire de Charles Huber figure aux p. 147-148.

15- Note sur son projet de voyage en Arabie, s. d. (avant 1878), 6 p., document d'archives, département des Cartes et plans de la Bibliothèque nationale de France, collections de la Société de géographie.

16- À propos de Julius Euting, la bibliographie est abondante. Citons notamment, en français, Christophe Didier, « Portrait d'un fondateur : Julius Euting », in La Revue de la BNU, ${ }^{\circ} 2$, 2010 p. 104-115 ; idem, « Euting, Julius », in Dictionnaire culturel de Strasbourg (1830-1880), Strasbourg, Presses universitaires de Strasbourg, 2017, p. 184-185.

17- Euting, Julius, Tagebuch einer Reise in Inner-Arabien, Leiden, Brill, 1896 (vol. 1) et 1914 (vol. 2). Réédité en 2004 chez Olms. 


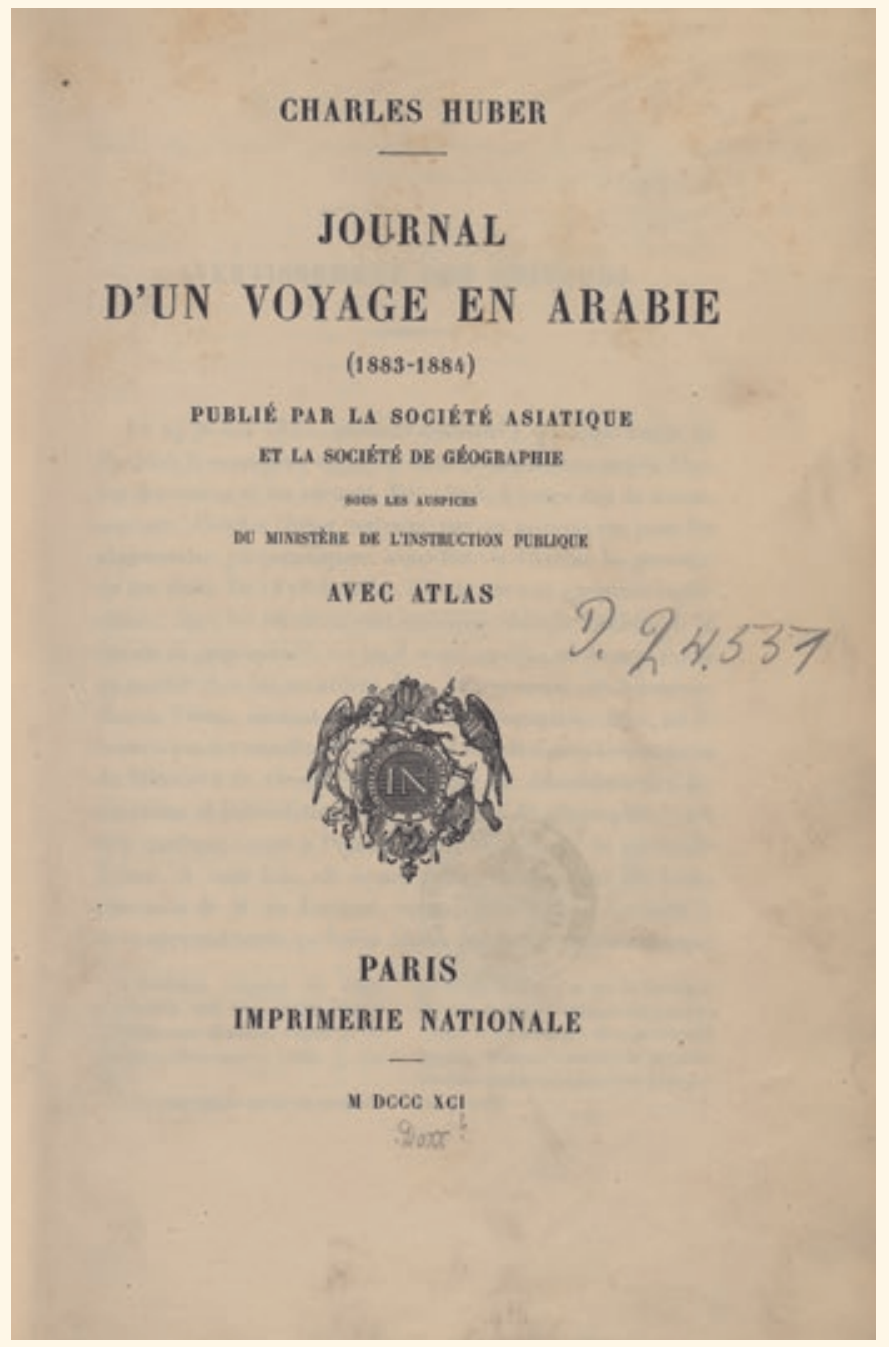

L'édition du Journal d'un voyage en Arabie

(1891; coll. BNU)

18- Huber, Charles, Journal d'un voyage en Arabie, 1883-1884, publié par la Société asiatique et la Société de géographie, sous les auspices du ministère de l'Instruction publique, avec atlas, Paris, Imprimerie nationale, 1891 ; idem, « Voyage dans l'Arabie centrale... », s. d. (218 p.), publié dans le Bulletin de la Société de géographie, $7^{\mathrm{e}}$ série, 5 (1884), p. 304-363, 468-530, et 6 (1885), p. 92-148. Charles Huber envoie des informations sur son voyage au Proche-Orient (Djeddah, 20 juin 1884), partiellement publiées dans les Comptes rendus des séances de la Société de géographie, 1884, p. 433 [document d'archives, 20 juin 1884].

19- «Voyage dans l'Arabie centrale », op. cit., 1884, p. 490-491

20- Charles Montagu Doughty (1843-1926). Écrivain, poète et grand voyageur, il est surtout connu pour son récit de voyages en deux volumes, Travels in Arabia Deserta (1888).

21- Sur le clan al-Shiyābīn de la tribu 'Utaybah, voir : Kurpershoek, Marcel, Oral Poetry and Narratives from Central Arabia, vol. 3, Bedouin Poets of the Dwāsir Tribe. Between Nomadism and Settlement in Southern Najd, 1999, p. 46 et 88 : « al-Shiyābīn' des 'Utaybah, une tribu notoirement connue à travers le Najd pour ses combattants féroces et leur indiscipline bédouine ».
22- Sur les objets de la mission Huber en Arabie ramenés en France et déposés au musée du Louvre, voir : Calvet, Yves et Robin, Christian, Arabie heureuse, Arabie déserte. Les antiquités arabiques du musée du Louvre, Paris, Éditions de la Réunion des musées nationaux, 1997.

23- Lozachmeur, Hélène et Briquel-Chatonnet, Françoise, " Charles Huber und Julius Euting in Arabien nach französischen, auch heute noch nicht veröffentlichten Dokumenten », in Anabases, $n^{\circ}$ 12, 2010, p. 195-200 : « Tu apprendras donc avec satisfaction qu'il a pu arriver jusqu'à Haill, mais qu'une fois là j'ai obtenu de l'émir de le faire filer par l'ouest ».

24- Ibid.

25- Ibid.

26- «Voyage dans l'Arabie centrale », op. cit., 1885, p. 123-124

27- Inscriptions lihyanites : provenant de l'ancien royaume de Lihyan. Voir aussi Christophe Didier, « Portrait d'un fondateur : Julius Euting ", in La Revue de la BNU, n² 2, 2010, partic. p. 115, note 7 .

28- Coufique : style de calligraphie arabe d'aspect angulaire, originaire de la ville de Koufa, dans l'actuel Irak. 